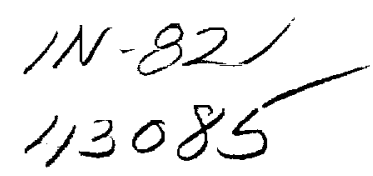

\title{
Coping with Data from Space Station Freedom 29
}

\author{
Marjory J. Johnson
}

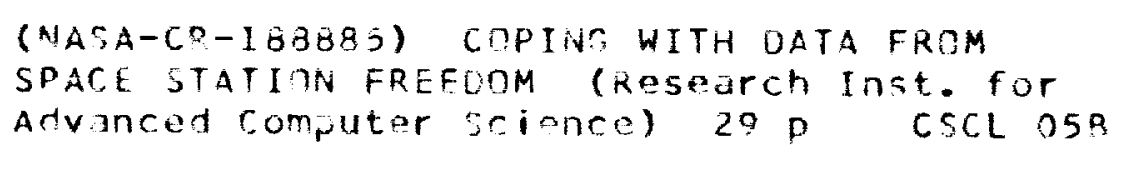

N91-33005

SPACE STATION FREFUOM (RESEarCh InSt. for
Unclis
0043095

RIACS Technical Report 91.05

January 1991

To appear in Computer Networks \& ISDN Systems 
$=$. 


\title{
Coping with Data from Space Station Freedom
}

\author{
Marjory J. Johnson
}

The Research Institute for Advanced Computer Science is operated by Universities Space Research Association, The American City Building, Suite 311, Columbia, MD 21044, (301)730-2656

Work reported herein was supported joinuly by the National Aeronautics and Space Administration (NASA) and the Defense Advanced Research Projects Agency (DARPA) under Cooperative Agreement NCC 2-387 to the Universities Space Research Association (USRA). 


$$
\text { . }
$$




\title{
Coping with Data from Space Station Freedom
}

\author{
Marjory J. Johnson \\ RIACS \\ NASA Ames Research Center \\ Moffett Field, California 94035 \\ U.S.A.
}

\begin{abstract}
The volume of data from future NASA space missions will be phenomenal. In this paper we examine the expected data flow from the Space Station Freedom and describe techniques that are being developed to transport and process that data.
\end{abstract}

\section{Introduction}

Future space missions such as Space Station Freedom and Mission to Planet Earth will exacerbate an already serious data-handling problem within NASA. Both of these missions are complex, involving multiple spacecraft, a broad user community, and thousands of scientific instruments. Besides the large number of instruments that will be collecting data to transmit to the ground, the data rates of some of the new highresolution observational instruments will be very high, e.g., 100 Mbps for Landsat-4, 300 Mbps for SAR (Synthetic Aperture Radar), and as high as 450 Mbps for HIRIS (High Resolution Imaging Spectrometer).

NASA has projected that by 1995 the volume of telemetry data from space-based scientific instruments will be on the order of 3 gigabits per second [12]. Not only is this orders of magnitude more data than can be transmitted from space to ground using current institutional facilities, but current ground-based networks are incapable of delivering such an enormous volume of data to the scientific community once it reaches the ground. 
Space Station Freedom will be the first example of an advanced orbiting system, a system so complex that it requires extensive on-board networking, extensive datahandling facilities on the ground, and the ability to support space-to-ground data rates as high as hundreds of megabits per second. The purpose of this paper is to describe the Space Station Freedom networking environment and to characterize data requirements for the end-to-end communications system.* We emphasize the space-to-ground data requirements, since these requirements will help to drive future high-speed networking both in space and on the ground.

\section{Networking in Space}

Networking in space poses unique problems. NASA, as well as other space agencies, has developed some general approaches to cope with the anomalies of space networking. Both the problems and general approaches to coping with them are addressed in the first subsection below.

Because of the expense of developing unique data-handling solutions to support each individual space mission, NASA is currently developing both standardized protocols and institutional facilities that will provide general support for space missions of the Space Station era. The last three subsections present aspects of this institutional support structure.

\subsection{Uniqueness of Environment}

Some of the problems associated with space networking are obvious, e.g., the unavoidable space-to-ground transmission delay and the stringent limitations on available

* Information contained herein reflects current (fall 1990) plans for the Space Station and for the NASA institutional facilities which will support space-to-ground communications in the Space Station era. This information is subject to change as the design of the various facilities progresses. 
resources, including on-board resources (power, weight, and volume), space-to-ground bandwidth, and ground processing facilities. Such problems often necessitate the development of specialized protocols to support space applications. This topic will be addressed further in Section 2.3.

Both to cope with resource constraints and to ensure mission safety and reliability, tight control is exercised over all mission activities. The flexibility provided to the end user of a general-purpose ground-networking system is not permitted for space applications. The use of all resources is tightly scheduled, from crew activities, to specification of resource envelopes for each payload, to the precise times that specific instruments will be turned on, to partitioning of the space-to-ground channel for transmission of various types of data.

Another general problem that must be addressed for all space applications is the lack of constant communication with the ground. There are several reasons for this: the fact that unmanned space platforms are typically scheduled for only a 10- to 20-minute space-to-ground communications window per 90-minute orbit, the lack of sufficient space-to-ground channel capacity to transmit all the data in real time, and the presence of a small zone of exclusion during each spacecraft orbit when ground communication is impossible.* Accordingly, much of the data that will be transmitted from space to ground is recorded and stored on-board, for transmission during a later communications window.

This recording of data introduces artifacts that must be removed once the data reaches the ground. Currently the recording media is tape; to save wear both on the tapes and on the recorders, the data is transmitted to ground in reverse order. To ensure that no

\footnotetext{
*Even though manned spacecraft will be allowed almost constant communication with ground, they are nevertheless subject to the zone of exclusion.
} 
data is lost, there is some overlap in data that is transmitted in real time and data that is recorded. Hence, preliminary processing on the ground (called level-zero processing within NASA) is required to reverse the recorded data, remove overlaps between realtime and recorded data, and resequence the data before it is transmitted to the end user.

Finally, data delivery to the end user often takes several days. Because of the large volumes of data involved, the bulk of the data from space is stored temporarily at specified data-handling facilities and delivered to the end user at his convenience (probably during off-peak hours, to reduce the cost). The availability of high-speed, costeffective ground networks might significantly change the nature of space networking.

\subsection{Tracking and Data Relay Satellite System (TDRSS)}

The Tracking and Data Relay Satellite System is NASA's primary data transport system for relaying data between earth-orbiting spacecraft and the ground; it is an institutional resource that will be shared by multiple NASA space missions, including Space Shuttle, Hubble Space Telescope, Space Station Freedom, and Mission to Planet Earth. TDRSS currently consists of three communications satellites in geosynchronous orbit, 22,500 miles above the earth; a fourth satellite will be added to the system in the future. Each satellite has two 150 megabit-per-second space-to-ground channels (the I and $Q$ channels), for a total downlink capacity of 300 megabits per second; the ground-to-space channel is 25 megabits per second. These satellites will be arranged in two sets, one located over the Pacific Ocean and the other located over the Atlantic Ocean, in order to provide almost continual coverage of spacecraft in low-earth orbit. The zone of exclusion for Space Station Freedom, when it will be out of the line of sight of either set of TDRSS satellites, will be approximately 10 minutes during each 90 -minute orbit. 


\subsection{Consultative Committee for Space Data Systems (CCSDS) Recommendations}

As an outgrowth of collaborative efforts between NASA and the European Space Agency (ESA), the Consultative Committee for Space Data Systems was formed in 1982 [9]. Recognizing the benefits of developing common solutions to data-handling problems, major space agencies throughout the world now belong to the organization. The charter of CCSDS is to develop recommendations for data-system standards to support space missions. Although these recommendations are not considered binding on any of the member agencies, adherence to the recommendations will provide compatibility of data-handling systems among cooperating agencies. This will facilitate collaborative space missions such as Space Station Freedom.

The Consultative Committee for Space Data Systems has recently developed a Recommendation for Advanced Orbiting Systems (AOS) [3,4,9], the first example of which will be Space Station Freedom. A major part of the Recommendation specifies services and data formats for transmission of data over the space-link subnet, i.e., TDRSS. Efficient use of bandwidth on the space-link subnet was a primary driver for development of the AOS Recommendation.

Since it is impossible using current technology to process data at the rate of 150 megabits per second, the AOS Recommendation is based on the use of virtual channels. A single physical space-to-ground channel is shared by multiple virtual channels. Transmission over the space-link subnet is patterned after time-division multiplexing, which is generally considered to provide the highest channel utilization in a heavily loaded network. Fixed-length data blocks from different virtual channels are interleaved on the physical space channel; consecutive data blocks are separated by 32-bit synchronization markers. There is a single data-block length for all virtual channels that share the same physical space channel. The format of this data-block is described in Section 4.2. 
Fill data is transmitted if necessary, so that data is transmitted over the physical space channel as a synchronous symbol stream, with synchronization markers appearing at constant intervals. This transmission pattern facilitates simple, robust synchronization processes at the ground terminus.

Several services have been defined within the AOS Recommendation, to satisfy all data-handling requirements that are anticipated for space missions during the Space Station era. Only a subset of these services has been selected for Space Station Freedom Program use.

\subsection{NASA Institutional Ground Support}

In the past NASA has developed separate ground data-handling facilities to support each individual space mission. As a cost-effective measure to handle the enormous volumes of data that are expected in the future, NASA is developing an institutional ground data-handling facility, called the Customer Data and Operations System (CDOS), to support multiple NASA space missions of the Space Station era, beginning in the mid 1990s [11]. Data transmission rates that will be handled by CDOS will challenge current communication technologies; high-rate data (i.e., $20 \mathrm{Mbps}$ to $300 \mathrm{Mbps}$ ) is expected to account for $80 \%$ of the data flow within the system.

The Space Station Freedom will be the first mission supported by CDOS. Functions that CDOS will provide include mission command and control, mission management, data handling (e.g., level-zero processing and storage of data), data distribution, and data routing. In this paper we are interested only in the data-distribution and data-routing functions.

At present NASA has only one ground terminal, located at the White Sands Complex (WSC) in White Sands, New Mexico. All data from TDRSS is transmitted to the 
WSC. A second ground terminal, which will also be located at White Sands, will be added in the Space Station era. Because of the cost of handling high data rates, the 300 megabit-per-second data stream (i.e., the two 150 megabit-per-second streams on the I and $Q$ channels) from TDRSS will be demultiplexed as early as possible into lower-rate data streams. This is accomplished by the Data Interface Facility (DIF), which will separate the data into individual virtual channels. The DIF, which is also located at White Sands, is being designed to handle at least four $300 \mathrm{Mbps}$ data streams simultaneously; the total volume of data handled by the DIF is expected to exceed 1000 gigabytes per day.

The NASA Communications (NASCOM) organization [13] provides institutional wide-area networking facilities for the transmission of mission-critical data between White Sands and various mission-control centers located throughout the United States. NASCOM is currently designing a network to satisfy the higher data-rate (up to 150 megabits per second), data-volume, and data-quality requirements of NASA space missions of the Space Station era. The NASCOM network will interface with the DIF at White Sands. Data from the DIF will be routed via NASCOM to appropriate first-level destinations, depending on the type of data transmitted on the virtual channel and hence the type of data handling required. Both the Space Station Control Center (SSCC) at NASA Johnson Space Center (Houston, Texas) and the Payload Operations Integration Center (POIC) at NASA Marshall Space Flight Center (Huntsville, Alabama) will be first-level destinations. Space Station operations data (i.e., core data) will be sent to the SSCC and data to manage scientific operations will be sent to the POIC. Facilities maintained by NASA's international partners may also be first-level destinations. Other NASA centers, such as Goddard Space Flight Center (GSFC) located in Greenbelt, Maryland (which provides data-handling services for scientific users), are likely to be included 
as nodes on the NASCOM network. Data will be transmitted from the NASCOM nodes to the end user (commercial customers, intemational partners, NASA or NASAsupported experimenters, other government agencies, or various control centers) via either public or private networks. High-rate payload data may be sent directly from the DIF to the payload user, possibly via domestic satellite links.

\section{Space Station Freedom System}

In this section we give an overview of the Space Station Freedom System.

\subsection{System Configuration}

Space Station Freedom will be a permanently manned international space facility, a constellation of spacecraft in low-earth orbit (220 miles high) with the U.S. manned base as its hub. It is scheduled to be on-orbit in its initial configuration in the mid 1990's. Assembly of the Space Station will continue through 1999. Initially it will be mantended; it is scheduled to become permanently manned in 1997. The system will continuously evolve during its expected thirty-year lifetime. In addition to the U.S. manned base, the Space Station Freedom System will include co-orbiting platforms, orbitaltransfer vehicles, orbital-maneuvering vehicles, extravehicular maneuvering units, and pressurized modules provided by the European Space Agency (ESA) and the National Space Development Agency (NASDA) of Japan.

\subsection{System Objectives}

The Space Station Freedom System will serve as a scientific laboratory, as a manufacturing facility, and as a base for deep-space exploration.

Scientific payloads will be located on the co-orbiting platforms as well as within the pressurized modules. A variety of scientific experiments will be conducted [10]. Pay- 
loads that probably will produce the highest data rates will be observational instruments, making observations of the earth, the ocean, and the atmosphere. Biological experiments will include microbial cultivation; micro/macrobial plant and animal development, reproduction, adaptation, and regeneration; and study of the effects of radiation on biological systems. Examples of physics and chemistry experiments will include materials processing, crystal growth, emulsion and chemical-mixing processes, fluid flow, and the effects of both high and low temperatures on physical and chemical systems. These experiments may provide valuable information that will lead to the development of manufacturing techniques in the space environment for ultra-pure materials that are difficult or impossible to produce on earth. Experiments to determine how well human beings can adapt to living in the space environment include monitoring of vital human systems during controlled exercise (e.g., bicycling), development of medical techniques for use in low gravity, and measurement of psychological stress.

The Space Station Freedom will serve as a repair center for orbiting platforms and satellites, just as the Space Shuttle has in the past. In its role as a base for further space exploration, Space Station Freedom may serve as home base during construction of a manned lunar base. It may also serve as a base for exploration of the planet Mars.

\subsection{Communications System Architecture}

Because of the massive volumes of data which must be transported on-board, on the ground, and from space to ground, high-speed data-handling capabilities are needed in all three locations.

Traditionally space communications systems have been designed conservatively, to satisfy safety and reliability requirements. The MIL-STD-1553B command/response multiplex bus [1] has been used, because it provides deterministic behavior for control of critical operations. Designers of the Space Station Freedom Data Management System 
recognize that greater flexibility and enhanced performance are required for the system. Yet, the introduction of distributed systems into space-mission communications has an associated risk. Inability to control the timing of interactions between distributed elements causes a distributed system to exhibit somewhat unpredictable behavior, unlike the command/response behavior of the MIL-STD-1553B bus. Hence, the use of distributed systems is being approached conservatively for the Space Station Freedom Data Management System (DMS). The DMS will utilize local-area networking technology. However, while subsystems are physically distributed around the network, there is little functional interaction between them.

There are two distinct types of users for Space Station communications facilities. Core users will be responsible for the health and safety of the spacecraft itself, while payload users will conduct experiments in the unique environment provided by space. A 100 megabit-per-second FDDI token ring [7], called the DMS optical network, will serve as a backbone local area network on-board the Space Station. Scientific payloads, sensors and effectors to monitor and control spacecraft operations, and general-purpose processing and storage facilities will all be connected to the DMS optical network. An earlier decision to connect the sensors/effectors and some of the low-rate payloads to the backbone via MIL-STD-1553B buses is being reconsidered because the 1 megabit-per-second data rate is likely to be inadequate; 10 megabit-per-second IEEE 802.4 token buses may be used instead. In the initial configuration of Space Station core data (i.e., data to control spacecraft operations) and payload data (i.e., both telemetry data from the scientific instruments and data to command and control the payloads) will share networking resources. In the assembly-complete configuration (scheduled for 1999) core data will be separated from payload data, thus ensuring that payload data will not interfere with critical spacecraft operations, by splitting the FDDI backbone into two FDDI networks con- 
nected by a bridge. One FDDI network will serve as a core network; the other FDDI network will serve as a payload network.

The Communications and Tracking (C\&T) System on-board the U.S. manned base is the single communications interface between the Space Station Freedom System and the ground. It is here that data is formatted and assigned to virtual channels. Then, using an appropriate release algorithm, data units from different virtual channels will be multiplexed into two 150 megabit-per-second data streams for transmission over the TDRSS physical channels. C\&T will also be the distribution point for all data that is transmitted from ground to space.

Command and control of all payloads will be accomplished via the DMS optical network. However, only low-data-rate payloads will use the DMS optical network for transmission of telemetry data to the C\&T interface, since the network-interface unit is not required to handle more than 10 megabits per second. Payloads whose data rates exceed 10 megabits per second will be connected directly to C\&T, using dedicated point-to-point fiber-optic links, called High-Rate Data Links. Telemetry data will be routed over these dedicated links. Similarly, High-Rate Data Links will be used for transmission of high-rate video data and to provide downlink service for data from the international partners. These High-Rate Data Links will initially handle up to 100 megabits per second; in evolutionary stages of the Space Station, they will handle up to a gigabit per second. Note that because of the 10-megabit-per-second limitation imposed by the network-interface unit for the DMS optical network, the High-Rate Data Links provide the only means for high-speed data transmission on-board the Space Station, at least in its assembly-complete configuration. The DMS optical network and the networkinterface unit may be upgraded as the system evolves during its thirty-year lifetime. 
The space-to-ground link is provided by the Tracking and Data Relay Satellite System (TDRSS), the NASA institutional facility described in Section 2.2, using the CCSDS Advanced Orbiting System Recommendation described in Section 2.3. Space Station Freedom has been allocated the full capability of one satellite, i.e., 300 megabits-persecond bandwidth for downlink transmission and 25 megabits-per-second bandwidth for ground-to-space transmission.

The ground terminal for TDRSS communications is located at White Sands, New Mexico. Space Station Freedom data will be serviced by the Customer Data and Operations System (described in Section 2.4) before it is distributed to the end user, using a combination of NASA institutional networks and public networks.

Figure 3.1 presents the end-to-end communications system architecture for Space Station Freedom, illustrating the virtual channels flowing into C\&T*, and being split out at the Data Interface Facility. Data from different virtual channels will be sent to different destinations, depending on the type of handling that is required for the data. By immediately splitting the high-rate data stream from space to ground into lower-rate virtual channels, it will be possible to handle the expected large volume of data using current technology.

*Some of the data enters the C\&T as virtual channels, while other data is actually formatted into virtual channels within the C\&T. Figure 3.1 does not illustrate internal details of any of the communications subsystems. 


\section{TDRSS}

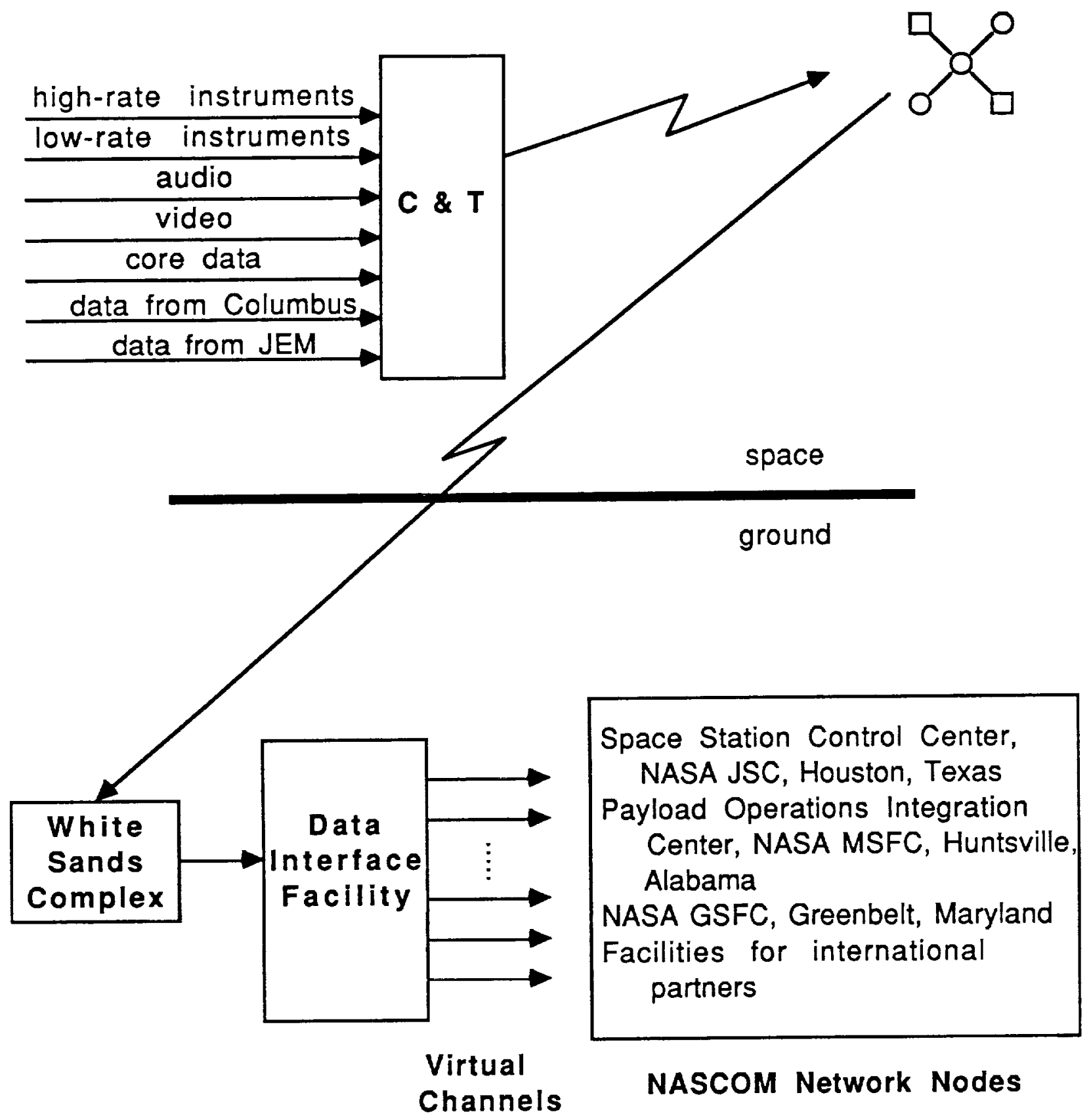

Figure 3.1. Space Station Freedom End-to-End Communications Architecture 


\section{Data Requirements for Space Station Freedom}

Communications will be required to monitor and control spacecraft operations, to monitor and control the scientific payloads, and to transmit scientific data to the ground.

\subsection{Data Types}

Table 4.1 describes the principal data types that will be used for Space Station Freedom.*

Telemetry data, i.e., measurement data from scientific instruments located in space, will account for approximately $80 \%$ of the data that will be transmitted from Space Station Freedom to the ground. There will be a variety of payloads, with widely varying data rates. Some data will also be required for continual monitoring of payload activities.

Core data is data associated with command and control of the spacecraft. Several critical subsystems have been defined, each to control a different resource, e.g., Electrical Power System, Thermal Control System, Environmental Control and Life Support System, and Fluid Management System. Thousands of sensors will monitor the operations of these subsystems, measuring temperature, pressure, flow, etc. Sensor readings will be transmitted to the ground, where final control of the Space Station Freedom System resides. Critical system parameters will be controlled from the ground via commands to effectors.

\footnotetext{
*Tables 4.1 and 4.2 are taken from [5].
} 
Table 4.1. Principal Data Types for Space Station Freedom

\begin{tabular}{|c|c|}
\hline Data Type & Description \\
\hline ASCII text & $\begin{array}{l}\text { Electronic mail, flight plans, procedures, } \\
\text { text file transfers (core or payload, } \\
\text { bidirectional) }\end{array}$ \\
\hline Conference video & $\begin{array}{l}\text { Video for routine space/ground discussions } \\
\text { and payload control, recreation, etc. } \\
\text { (core or payload, bidirectional) }\end{array}$ \\
\hline Conference audio & $\begin{array}{l}\text { Audio for routine space/ground } \\
\text { discussions and payload control, } \\
\text { recreation, etc. (core or payload, } \\
\text { bidirectional) }\end{array}$ \\
\hline Operational video & $\begin{array}{l}\text { Full resolution video for observing critical } \\
\text { activities (core or payload, bidirectional) }\end{array}$ \\
\hline Operational audio & $\begin{array}{l}\text { High-quality audio for discussing critical } \\
\text { activities (core or payload, bidirectional) }\end{array}$ \\
\hline Operational telemetry & $\begin{array}{l}\text { Engineering telemetry, monitoring critical } \\
\text { activities (core or payload) }\end{array}$ \\
\hline Operational command & $\begin{array}{l}\text { Engineering telecommands, controlling } \\
\text { critical activities (core or payload) }\end{array}$ \\
\hline Computer or database load & $\begin{array}{l}\text { Exchange of data between computer } \\
\text { memories (core or payload, bidirectional) }\end{array}$ \\
\hline $\begin{array}{l}\text { High-rate payload digital } \\
\text { science - } \\
\text { Bulk: } \\
\text { Quick look: }\end{array}$ & $\begin{array}{l}\text { Imaging telemetry - } \\
\text { Offline analysis, research-oriented } \\
\text { Near-real-time analysis }\end{array}$ \\
\hline $\begin{array}{l}\text { Low to medium rate } \\
\text { payload digital science - } \\
\text { Bulk: } \\
\text { Quick look: }\end{array}$ & $\begin{array}{l}\text { Non-imaging telemetry - } \\
\text { Offline analysis, research-oriented } \\
\text { Near-real-time analysis }\end{array}$ \\
\hline Text and graphics hardcopy & $\begin{array}{l}\text { High-fidelity pixel map of digitized image } \\
\text { (e.g., fax) for planning, troubleshooting, } \\
\text { recreation or discussions (core or payload, } \\
\text { bidirectional) }\end{array}$ \\
\hline Interactive query & $\begin{array}{l}\text { Discrete messages transmitted to remote } \\
\text { application process to initiate subroutines, } \\
\text { for data base access, etc. (bidirectional) }\end{array}$ \\
\hline Bulk data & $\begin{array}{l}\text { Bulk transfer of general-purpose digital } \\
\text { data, e.g., low fidelity imagery, } \\
\text { newspapers (bidirectional) }\end{array}$ \\
\hline
\end{tabular}


In addition to being used for recreational, conferencing, and public-relations purposes, two-way voice and video enhance operational capabilities for both core and payload activities. For example, audio and video are indispensable during delicate docking maneuvers. Also, during an extra-vehicular activity (EVA), audio and video communications between the crew members performing the EVA and crew members inside the spacecraft will greatly facilitate the activity.

Two-way audio and video communications may also improve the quality of science that is conducted on-board Space Station Freedom by enabling ground-based scientists to interact with their on-board experiments. This mode of operation is called telescience. A study conducted recently at NASA Ames Research Center examined the benefits of using two-way audio and video to allow a scientist at his home institution to monitor a crew member's activities as he worked with plant specimens, while the crew member in turn watched the scientist perform the same activities on the ground. The remote coaching provided by the ground-based scientist appeared to have significant benefits [8].

The other types of data listed in the table, such as electronic mail, data-base access, and file transfer, serve the same general-purpose functions as in any communications system.

\subsection{Data Formats}

Use of the newly developed CCSDS Advanced Orbiting Systems data formats has been specified for Space Station Freedom. As stated in Section 2.3, data will be transmitted over TDRSS using virtual channels. Different data types, such as audio, video, packet data, etc., will be placed in different virtual channels, to facilitate data handling on the ground. Different, traditionally conflicting, services can be provided for data on different virtual channels, e.g., high throughput for high-rate telemetry data and low delay for near-real-time command and control of the spacecraft or payloads. 
The AOS Recommendation specifies services for a broad range of space missions, rather than being directed explicitly toward the Space Station. Hence, many of the data-format options that are provided by the Recommendation will not be implemented for the Space Station, but may be used for other missions of the Space Station era. For this reason we first discuss the basic data formats provided by the Recommendation, and then we indicate which of these formats will be used for the Space Station.

The unit of transmission over the space-link subnet, as specified by the CCSDS AOS Recommendation, is either the Virtual Channel Data Unit (VCDU) or the Coded Virtual Channel Data Unit (CVCDU), depending on the grade of service that is required for the data being transmitted. A CVCDU is basically a VCDU with a block of ReedSolomon check symbols appended to provide a high degree of forward-error correction. Hence, use of a coded virtual channel provides the higher quality of service. The VCDUs (CVCDUs) are fixed-length data blocks; the length selected for Space Station Freedom is 10200 bits. The format of a VCDU and CVCDU is illustrated in Figure 4.1. The data field of the VCDU (CVCDU) consists of either a fixed-length bitstream or a fixed-length string of CCSDS packets multiplexed together to form the correct length data unit. 


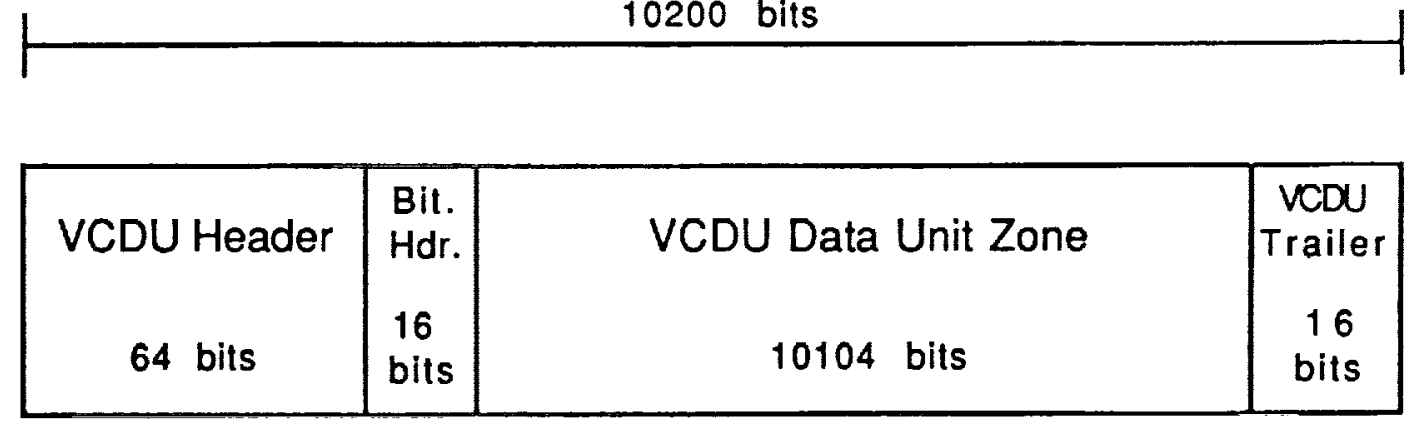

\section{Virtual Channel Data Unit (VCDU)}

\begin{tabular}{|c|c|c|c|}
\hline VCDU & $\begin{array}{c}\text { Bit.l } \\
\text { Mult. }\end{array}$ & VCDU Data Unit Zone & $\begin{array}{c}\text { Reed-Solomon } \\
\text { Check } \\
\text { Header }\end{array}$ \\
$\begin{array}{c}\text { Hdr. } \\
48 \text { bits }\end{array}$ & $\begin{array}{c}16 \\
\text { bits }\end{array}$ & 8856 bits & 1280 bits \\
\hline
\end{tabular}

Coded Virtual Channel Data Unit (CVCDU)

Figure 4.1. Structure of $V C D U$ and $C V C D U$ 
Packetized data will be formatted as CCSDS packets, which are multiplexed together to form the data field of a CVCDU.* The CCSDS packet format has been designed to minimize on-board processing and to maximize efficiency of transmission over TDRSS. Since CCSDS packet length may vary, the number of packets contained in a single CVCDU may also vary. In addition, it may be necessary to segment a packet in order to form the fixed-length data field of the CVCDU. The Multiplexing Data Unit header points to the beginning of the first full CCSDS packet contained in the data field.

The Recommendation specifies two different packet services, Path Service and Internet Service. Path Service, which uses only the CCSDS packet header, was designed for efficient transmission of telemetry data. Intemet Service, which is based on use of the full seven-layer stack of ISO/OSI (International Standards Organization/Open Systems Interconnection) network protocols, was designed for interactive use, such as electronic mail and file transfer. Internet packets must be encapsulated in CCSDS packets for transmission over TDRSS.

Bitstream Service is provided to handle data as an unstructured stream of bits. A bitstream from a single user is broken into blocks which will fit exactly into the fixedlength data field of the VCDU (CVCDU). In order to provide isochronous transfer for audio or video data, it may be necessary to release a VCDU (CVCDU) before the data field is completely filled. In this case fill data will be added. The Bitstream Data Unit header points to the end of the valid data in the data field.

The following formats will be used for Space Station Freedom [6]. All data will be Reed-Solomon encoded, so that only CVCDUs will be transmitted over TDRSS. Digitized video will be transmitted as packetized data, while digitized audio will be handled

\footnotetext{
* Packetized data must be transmitted in coded virtual channels, since the VCDU does not provide
} adequate error control to protect individual packet headers located within its data field. 
as a bitstream. Telemetry data may be transmitted using either Bitstream Service or Path Service.

\subsection{Data Volume}

TDRSS bandwidth allocations for Space Station Freedom are 300 megabits-persecond downlink and 25 megabits-per-second uplink. Since the volume of uplink data is relatively low, we don't discuss it further. Table 4.2 presents delivery characteristics of the principal data types given in Table 4.1. These statistics were collected as part of a Space Station planning activity during 1985 [5], so they should be regarded only as a general indication of requirements. The information in this table reveals two classes of data: high-volume telemetry data that has virtually no delay constraints and low-volume audio, video, and command-and-control data that does have delay constraints. There is miscellaneous traffic that doesn't fit into either category; the expected volumes, as designated in Table 4.2, are low.

Because the highest volume of data will be telemetry data, the total data flow from Space Station Freedom to the ground (excluding fill data) depends on the nature of the payloads that are flown. Payload rates will vary from less than $100 \mathrm{kbps}$ to several hundred megabits per second. At least initially, many of the instrument rates will be less than 1 megabit per second. Initially the High-Rate Data Links will carry up to 100 megabits per second. Eventually they will be upgraded to support data rates in the gigabit range. Various groups within NASA are compiling information about user requirements for Space Station Freedom. One database contains information about expected data rates for approximately 200 U.S., Canadian, Japanese, and ESA payloads [15]. Figure 4.2 is a histogram of the percentage of these payloads that fall within various data-rate categories. 
Table 4.2. Delivery Characteristics of Principal Data Types

\begin{tabular}{|c|c|c|c|}
\hline Data Type & Data Rate & $\begin{array}{l}\text { Maximum Transport } \\
\text { Delay }\end{array}$ & $\begin{array}{l}\text { Continuous } \\
\text { or Burst }\end{array}$ \\
\hline ASCII text & $<1 \mathrm{Mbps}$ & Minutes to hours & Burst \\
\hline Conference video & $\begin{array}{l}5 \text { channels } \\
5-10 \text { Mbps each }\end{array}$ & 1 to 2 seconds & Continuous \\
\hline Conference audio & $\begin{array}{l}5 \text { channels } \\
16-32 \text { kbps each }\end{array}$ & 1 to 2 seconds & Continuous \\
\hline Operational video & $\begin{array}{l}5 \text { channels } \\
<25 \text { Mbps each }\end{array}$ & 2 to 4 seconds & Continuous \\
\hline Operational audio & $\begin{array}{l}20 \text { channels } \\
32-64 \text { kbps each }\end{array}$ & 1 to 2 seconds & Continuous \\
\hline Operational telemetry & $<500 \mathrm{kbps}$ & 2 to 4 seconds & Continuous \\
\hline Operational command & $<100 \mathrm{kbps}$ & 2 to 4 seconds & Continuous \\
\hline Computer or database load & $<1 \mathrm{Mbps}$ & Minutes to hours & Burst \\
\hline $\begin{array}{l}\text { High-rate payload digital } \\
\text { science - } \\
\text { Bulk: } \\
\text { Quick look: }\end{array}$ & $\begin{array}{l}<300 \mathrm{Mbps} \\
<30 \mathrm{Mbps}\end{array}$ & $\begin{array}{l}\text { Days } \\
\text { Minutes }\end{array}$ & $\begin{array}{l}\text { Continuous } \\
\text { Burst }\end{array}$ \\
\hline $\begin{array}{l}\text { Low to medium rate } \\
\text { payload digital science - } \\
\text { Bulk: } \\
\text { Quick look: }\end{array}$ & $\begin{array}{l}<1 \text { Mbps } \\
<1 \text { Mbps }\end{array}$ & $\begin{array}{l}\text { Hours } \\
\text { Seconds }\end{array}$ & $\begin{array}{l}\text { Continuous } \\
\text { Burst }\end{array}$ \\
\hline Text and graphics hardcopy & $<1 \mathrm{Mbps}$ & Minutes & Burst \\
\hline Interactive query & $<10 \mathrm{kbps}$ & 2 to 4 seconds & Burst \\
\hline Bulk data & 1-5 Mbps & Minutes & Continuous \\
\hline
\end{tabular}




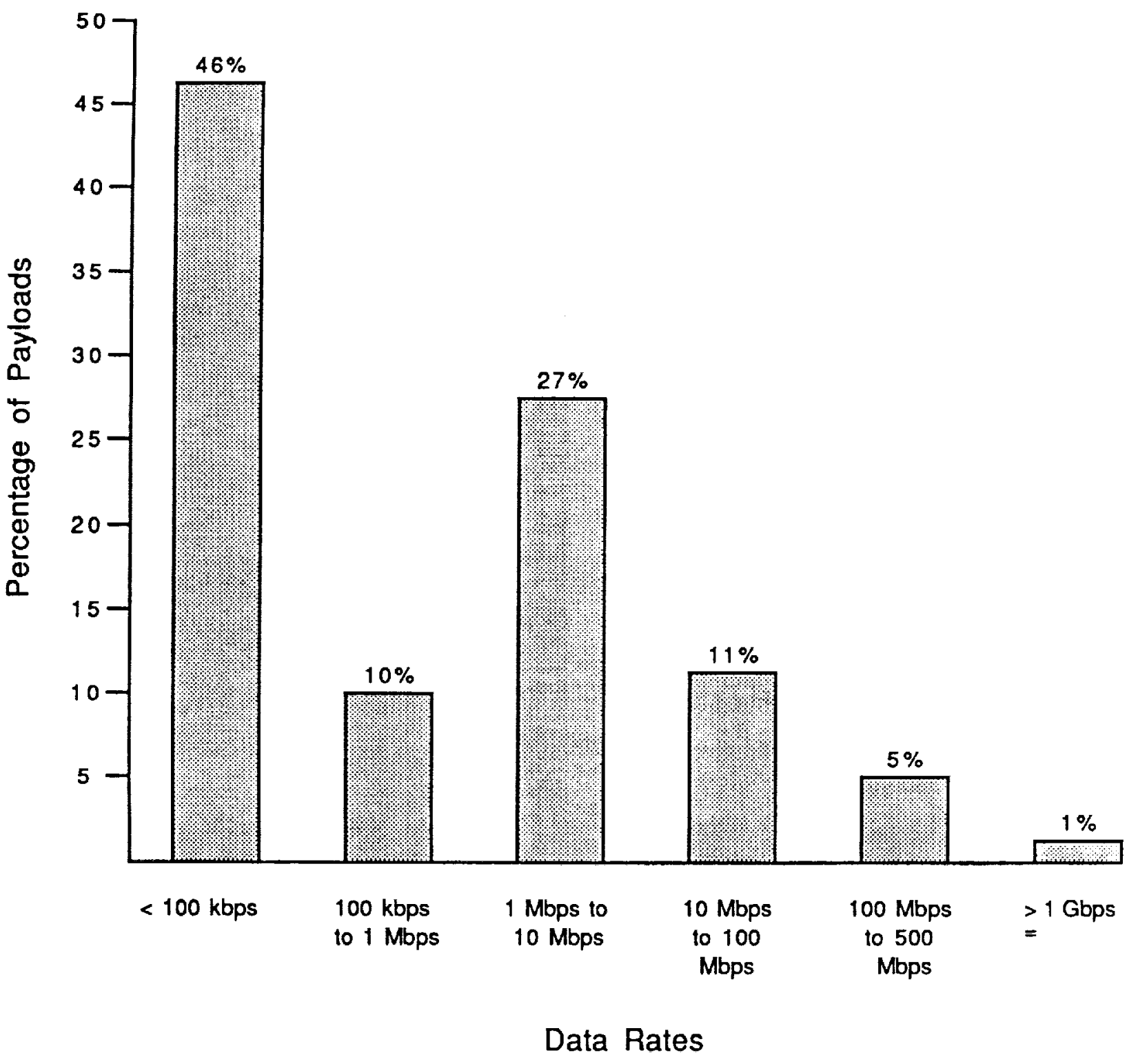

Figure 4.2. Percentage of Payloads at Each Data Rate 
Just as payload data rates vary, the size of a packet containing telemetry data also varies. The length ranges from approximately 500 octets to 2000 octets, depending on the type of instrument. The average size is approximately 1000 octets. Engineering packets, which contain diagnostic information, will be shorter, approximately 100 octets in length.

High-rate (i.e., higher than $10 \mathrm{Mbps}$ ) payloads transmit telemetry data via dedicated High-Rate Data Links physically connected to C\&T through a Patch Panel. Though in its assembly-complete configuration the Space Station will have hundreds of High-Rate Data Links distributed to payloads throughout the station, at most 8 high-rate payloads can be attached to the Patch Panel simultaneously. The crew must manually reconfigure the Patch Panel to change from one set of payloads to the next.

Initially the High-Rate Data Links will be able to transfer at most 100 megabits per second, so data from payloads with higher rates must be compressed. In order to grasp the true impact of telemetry data on the overall Space Station Freedom communications system, it is necessary to understand the nature of the telemetry-gathering process. Traditionally payload instruments are turned on, gathering data, according to a schedule. The High-Rate Data Link connections to the Patch Panel can be switched from one set of payloads to the next, according to this schedule. A single instrument may be turned on for minutes, hours, or even days at a time. During this time it is continuously generating bulk data, which will eventually be transmitted to the ground. Although the volume of this data may be enormous, it may be several days before the data is delivered to the end user. In addition to this continuous bulk data, a low volume of quick-look data is required to verify proper operation of the payload. Quick-look data is bursty, rather than continuous. 
The remaining data types that we discuss in this section must satisfy delay constraints of only a few seconds. The number of audio and video channels currently specified for the Space Station are close to the estimates listed in Table 4.2, though higher video rates will be available than anticipated. In the assembly-complete configuration of Space Station, there will be twenty-four simultaneous 64 kilobit-persecond audio channels, time-division multiplexed into the standard telephone $\mathrm{T} 1$ format at 1.544 Mbps [6]. Data rates supported for downlink video include $41 \mathrm{Mbps}, 21 \mathrm{Mbps}$, $11 \mathrm{Mbps}, 5 \mathrm{Mbps}, 1.5 \mathrm{Mbps}$, and a low-rate freeze-frame video [6]. Eight High-Rate Data Links will be dedicated to video.

Core data, including packetized data, audio, and video, is expected to be very low rate, only a few megabits per second. However, because of the critical importance of this type of data for safety and reliability, it is continuous in nature. Instrumentation data from sensors monitoring the status of the various Space Station Freedom critical subsystems will be multiplexed together before being transmitted over the FDDI network for eventual transmission to the ground.

Recall that the 300-Mbps TDRSS downlink data stream from Space Station Freedom will be split into lower-rate virtual channels as soon as it reaches the ground, so as to avoid the necessity of developing new technologies for a ground-based data-handling facility. Individual virtual channels will be routed to different locations, dependent on the type of data contained therein.

\section{System Evolution}

The nature of space missions is changing. Past missions, called conventional missions, have generated low-to-moderate data rates. Space Station Freedom, the first example of an adranced orbiting system, is expected to generate moderate-to-high data rates. As Space Station Freedom evolves over its expected thirty-year lifetime, the volume of 
its data flow is expected to become even larger. There are many reasons for the expected increase, including additional platforms, greater bandwidth capacity on-board the manned base, sophisticated payloads that generate data at increasingly higher rates, and more demand for interactive control of payloads by ground-based scientists. As an example of how payload data rates are expected to increase, initial earth-science instruments (1998 through 2004) will have an average rate of $26 \mathrm{kbps}$ and a peak rate of 66 kbps, while next-generation earth-science instruments are expected to generate data at an average rate of $13.3 \mathrm{Mbps}$ and a peak rate of $32 \mathrm{Mbps}$ [14].

Future missions following Space Station Freedom are expected to generate even higher quantities of data. For example, as part of Mission to Planet Earth, the U.S. plans to have two polar platforms in geosynchronous orbit by 1998 or 1999 . This mission, which will include many high-data-rate observational instruments, is expected to generate a trillion bits of data per day.

The expected downlink data volumes from these future space missions will strain TDRSS capacities. In fact there are indications that $300 \mathrm{Mbps}$ is insufficient bandwidth to support peak data flows from Space Station Freedom, even in its initial phases. Hence, it is essential to increase the capacity of NASA institutional space-to-ground links. Various approaches are being explored: use of point-to-point links to other places besides White Sands, addition of other ground stations, and development of higher-capacity communications satellites [2]. A more novel approach would be the processing of scientific data on-board the various spacecraft, to reduce the overall volume of data that must be transmitted to ground.

Ground data-handling capabilities must keep pace with the volumes of data that will be transmitted from space to ground. The Customer Data and Operations System will initially be able to handle 300 megabits per second (i.e., data from the Space Station 
Freedom). Its capacity will evolve first to 600 megabits per second and then to 900 megabits per second. The use of optical disks for storage of recorded data on-board the various spacecraft would ease the burden on ground data-handling facilities considerably, because it would eliminate the need for reversal of recorded data when it reaches the ground.

NASA is addressing another kind of evolution, as new methods of conducting space science are developed. NASA is committed to enabling scientists to have an increasing level of interaction with their space-borne experiments. While this mode of operation, i.e., telescience, presents exciting opportunities for scientists, extending their laboratories into space, it is a challenge for system developers to determine how to isolate core operations from possible interference (either intentional or unintentional) from payload users. Campaign-style missions, involving instruments on multiple spacecraft working together

on a single project, present further challenges. Appropriate communications capabilities are vital to the success of either of these modes of operation.

\section{Conclusion}

NASA's proposed solutions for handling the expected volume of data from Space Station Freedom are clearly a consequence of limitations of today's technology. Advances in high-speed networking would considerably facilitate the transfer of telemetry data from the space-based scientific instrument to the ground-based end user.

Advances in other technologies are also needed to enable the scientist to utilize all the data that he will receive. Currently storage rooms full of magnetic tapes contain telemetry data that has never been examined. The development of techniques for visualizing scientific data would help the scientist to comprehend the sheer volumes of data that are available to him. 


\section{References}

[1] "Aircraft Internal Time Division Command Response Multiplex Data Bus," MIL-STD-1553B, Notice 1, February, 1980.

[2] Daniel L. Brandel, William A. Watson, and Aaron Weinberg, "NASA's Advanced Tracking and Data Relay Satellite System for the Years 2000 and Beyond," Proceedings of the IEEE 78 (7) (1990), 1141-1151.

[3] Consultative Committee for Space Data Systems, "Advanced Orbiting Systems, Networks and Data Links: Architectural Specification," CCSDS 701.0-B-1: Recommendation for Space Data System Standards, Blue Book, Issue 1, October, 1989.

[4] Consultative Committee for Space Data Systems, "Advanced Orbiting Systems, Networks and Data Links: Summary of Concept, Rationale and Performance," CCSDS 700.0-G-2: Report Concerning Space Data System Standards, Green Book, Issue 2, October, 1989.

[5] Consultative Committee for Space Data Systems Report Concerning Space Data System Standards, "Space Station: Application of CCSDS Recommendations for Space Data System Standards to the Space Station Information System (SSIS) Architecture," Issue-1, 1985.

[6] C.R. Easton, J.F. Smith, "Data Services for Space Station Freedom," IEEE Network Magazine 4 (5) (1990), 29-32.

[7] FDDI Token Ring Media Access Control, American National Standard, X3.139 1987.

[8] Richard F. Haines, Vicki Johnson, Kristofer H. Vogelsong, and Walt Froloff, "Ames Life Science Telescience Testbed Evaluation," RIACS Technical Report \# 89.31, July, 1989.

[9] Adrian Hooke, "CCSDS Advanced Orbiting Systems: International Data Communications Standards for the Space Station Freedom," IEEE Network Magazine 4 (5) (1990), 13-16.

[10] Jet Propulsion Laboratory, Space Station Information System (SSIS) Final Study Report, JPL D-1737, August, 1984.

[11] NASA Goddard Space Flight Center, Customer Data and Operations System (CDOS) Concept Definition Document, August, 1988. 
[12] NASA Technical Memorandum 85631, NASA Computer Science Research Program Plan, 1983.

[13] Joseph F. Smith, Daniel Willett, and Sunil Paul, "Overview of the Space Station Communications Networks," IEEE Network Magazine 4 (5) (1990), 22-28.

[14] Summary Minutes: Space Station Science and Applications Advisory Subcommittee, Washington, D.C., December 6-8, 1989, published February 23, 1990.

[15] Supporting material for Space Station Freedom preliminary design review of the Data Management System, March, 1990. 